\title{
Zur Bezeichnung von Größen
}

Trotz verschiedener Autoren der einzelnen Teile wurde versucht, einheitliche Prinzipien bei der Bezeichnung der Größen durchzusetzen, wobei jedoch - bedingt durch die Originalarbeiten zum betreffenden Thema - kleinere Unterschiede verbleiben.

Fundamentale Konstanten $\left(\hbar, m, \epsilon, c, k_{\mathrm{B}}\right)$, atomare Einheiten $\left(a_{0}, r y d, 2 r y d\right)$ und charakteristische Größen des Elektronengases $\left(k_{\mathrm{F}}, q_{\mathrm{TF}}, \omega_{\mathrm{pl}}\right)$ s. [A 6].

Normierungsvolumen, Teilchenzahl

Teilchendichte

Spinvariable, Spinquantenzahl

Gesamtenergie, Einteilchenenergie

Zahl der Elektronenzustände pro Energieeinheit und pro

Elementarzelle bzw. pro Elementarzelle und Spin

Zahl der Elektronenzustände pro Energieeinheit und pro

Volumeneinheit bzw. pro Volumeneinheit und Spin

zugehörige integrierte Zustandsdichten

Phononenzustandsdichte

Gittergrößen :

Vektor im Gitter, im Gitter mit Basis

Auslenkung aus der Mittellage

Vektor im reziproken Gitter

Strukturfaktor

Bandindex

Zahl der Elementarzellen

Volumen pro Elementarzelle

Zahl der Atome pro Elementarzelle

mittleres Volumen pro Atom

zugehöriger Radius

Volumen der Wigner-Seitz-Zelle

zugehöriger Radius

Übergang von Summationen zu Integrationen

$$
\begin{aligned}
& v, N \\
& n=N / V \\
& \sigma, s \\
& E, \varepsilon \\
& D(\varepsilon), D_{s}(\varepsilon) \\
& \mathscr{D}(\varepsilon), \mathscr{D}_{s}(\varepsilon) \\
& N(\varepsilon), N_{s}(\varepsilon), \mathscr{N}(\varepsilon), \mathscr{N}_{s}(\varepsilon) \\
& g(\omega), F(\omega) \\
& \boldsymbol{R}, \boldsymbol{R}+\boldsymbol{s} \\
& \boldsymbol{u} \\
& \boldsymbol{G} \\
& S(\boldsymbol{q}), S(0)=1 \\
& v \\
& N \\
& v_{0}=\frac{v}{N} \\
& \text { M } \\
& v_{\mathrm{at}}=\frac{v_{0}}{M}=\frac{v}{M N} \\
& R_{\text {at }} \\
& v_{\mathrm{ws}} \\
& R_{\mathrm{WS}} \\
& \sum_{\boldsymbol{k}}=\int \frac{v \mathrm{~d}^{3} k}{(2 \pi)^{3}}
\end{aligned}
$$

